\title{
CO-EXISTENCE OF MEDULLARY AND PAPILLARY CARCINOMAS OF THYROID - CASE REPORT AND BRIEF REVIEW OF LITERATURE
}

\author{
Divya Narayanankutty ${ }^{1}$ Geetha Devadas ${ }^{2}$
}

${ }^{1}$ Registrar, Department of Haematology, Apollo Hospitals, Chennai.

${ }^{2}$ Professor, Department of Pathology, Madras Medical College, Chennai.

\begin{abstract}
BACKGROUND

Co-existence of medullary thyroid carcinoma (MTC) and papillary thyroid carcinoma (PTC) in the same thyroid is an extremely rare phenomenon. A few cases of such co-existence have been repored in literature. We report a case of 44-year-old lady who was detected to have thyroid swelling by her clinician, which on ultrasound was suggestive of multinodular goitre. Her thyroid function tests were normal. Fine needle aspiration cytology was suggestive of medullary carcinoma of thyroid. She underwent total thyroidectomy. Microscopically there were MTC foci on the right lobe with an adjacent foci of papillary carcinoma, both of which are separated by normal thyroid parenchyma. Left lobe also showed foci of PTC. Calcitonin, synaptophysin and chromogranin were positive in the MTC foci and thyroglobulin was positive in the PTC foci, confirming the diagnosis of coexistent MTC and PTC. Coexistent MTC and PTC is a rare clinical entity and should be considered in the differential diagnosis of thyroid tumours.
\end{abstract}

\section{KEYWORDS}

Medullary Thyroid Carcinoma, Papillary Thyroid Carcinoma, Co-existence.

HOW TO CITE THIS ARTICLE: Narayanankutty D, Devadas G. Co-existence of medullary and papillary carcinomas of thyroid - Case report and brief review of literature. J. Evolution Med. Dent. Sci. 2016;5(84):6296-6299, DOI: 10.14260/jemds/2016/1422

\section{BACKGROUND}

Simultaneous occurrence of medullary thyroid carcinoma (MTC) and papillary thyroid carcinoma (PTC) in the same thyroid is an extremely rare phenomenon and the surgeon and pathologist should be aware of such a possible coexistence. The incidence, cell of origin, histopathological features and prognosis are completely different for MTC \& PTC. PTC arises from thyroglobulin producing follicular cells, whereas MTC arises from calcitonin producing $\mathrm{C}$ cells. Tumours showing synchronous occurrence of MTC \& PTC have been reported in literature.[1-5] As per the available details, these tumours occurred together more commonly in women, in fifth to seventh decades of life, presented as palpable neck swellings and were treated by surgery with or without adjuvant therapy. ${ }^{[3]}$ Metastatic foci of either or both of the tumours were detected in a few patients. The exact pathogenesis of these concurrent tumours is unknown. Various hypotheses have been postulated to explain the possible pathogenesis which includes the stem cell theory, hostage hypothesis, field effect theory and collision theory. Genetic analysis of RET protooncogenes were done in various studies, but had conflicting results. Most of the reported cases were explained by collision theory, which suggests that two independent tumours are located in the same gland by simple coincidence. Our case could also be explained by this theory because of the presence of two tumours as separate masses, separated by normal thyroid parenchyma.

Financial or Other, Competing Interest: None.

Submission 13-06-2016, Peer Review 08-10-2016,

Acceptance 14-10-2016, Published 20-10-2016.

Corresponding Author:

Dr. Divya Narayanankutty,

No. 3B, Green Peace Fortuna,

Block No. 17,

P. T. Rajan Salai,

K. K. Nagar

Chennai.

E-mail: divyan_harisree@yahoo.co.in

DOI: $10.14260 /$ jemds/2016/1422

\section{CASE HISTORY}

A 44-year-old lady who is a known case of cholelithiasis, presented with complaints of colicky pain in right hypochondrium of three months duration. Clinician noticed a thyroid swelling of $3 \times 3 \mathrm{~cm}$ size which was firm in consistency. She gave no family history of thyroid malignancies or endocrinopathies. There was no history of radiation exposure. Serum values of triiodothyronine, tetraiodothyronine and thyroid stimulating hormones were within normal limits. Ultrasound neck showed multiple heteroechoic lesions, largest measuring $0.8 \times 0.7 \mathrm{~cm}$ in the right lobe of thyroid and was suggestive of multinodular goitre. Computed tomography of neck showed diffuse enlargement with altered density of both lobes of thyroid with speckled calcification in right lobe. There was no retrosternal extension or lymph node enlargement. Fine needle aspiration cytology was suggestive of medullary carcinoma. Whole body radiography revealed no bony abnormalities. Serum calcium levels were also normal. Total thyroidectomy was performed. Perioperatively, no lymph nodes were detected.

Grossly the right lobe of thyroid measured $4.5 \times 2 \times 2 \mathrm{~cm}$. Cut surface showed a calcified grey white nodule measuring $1.5 \mathrm{~cm}$ diameter. Another small nodule measuring $0.5 \mathrm{~cm}$ was seen in the lower pole, both nodules separated by normal thyroid parenchyma. Left lobe also showed two grey white nodules measuring $0.25 \mathrm{~cm}$ each. Microscopically sections from the larger nodule on right lobe showed a neoplasm composed of sheets, nests, cords and acinar configurations of cells with moderate eosinophilic cytoplasm, round to oval nuclei with stippled chromatin. Some foci showed spindled morphology of cells. Intervening stroma showed focal amyloid deposition and areas of calcification. Sections from smaller nodule in right lobe and both nodules in left lobe showed tumour cells dispersed in papillary configuration with central fibrovascular core. The cells had scant to moderate cytoplasm and optically clear nuclei showing crowding and focal grooves and pseudoinclusions. A diagnosis of medullary carcinoma with co-existent multicentric papillary carcinoma was given. 
MTC areas were strongly positive for calcitonin, synaptophysin and chromogranin and negative for thyroglobulin. PTC areas were positive for thyroglobulin and negative for calcitonin, synaptophysin and chromogranin confirming the histopathology diagnosis.
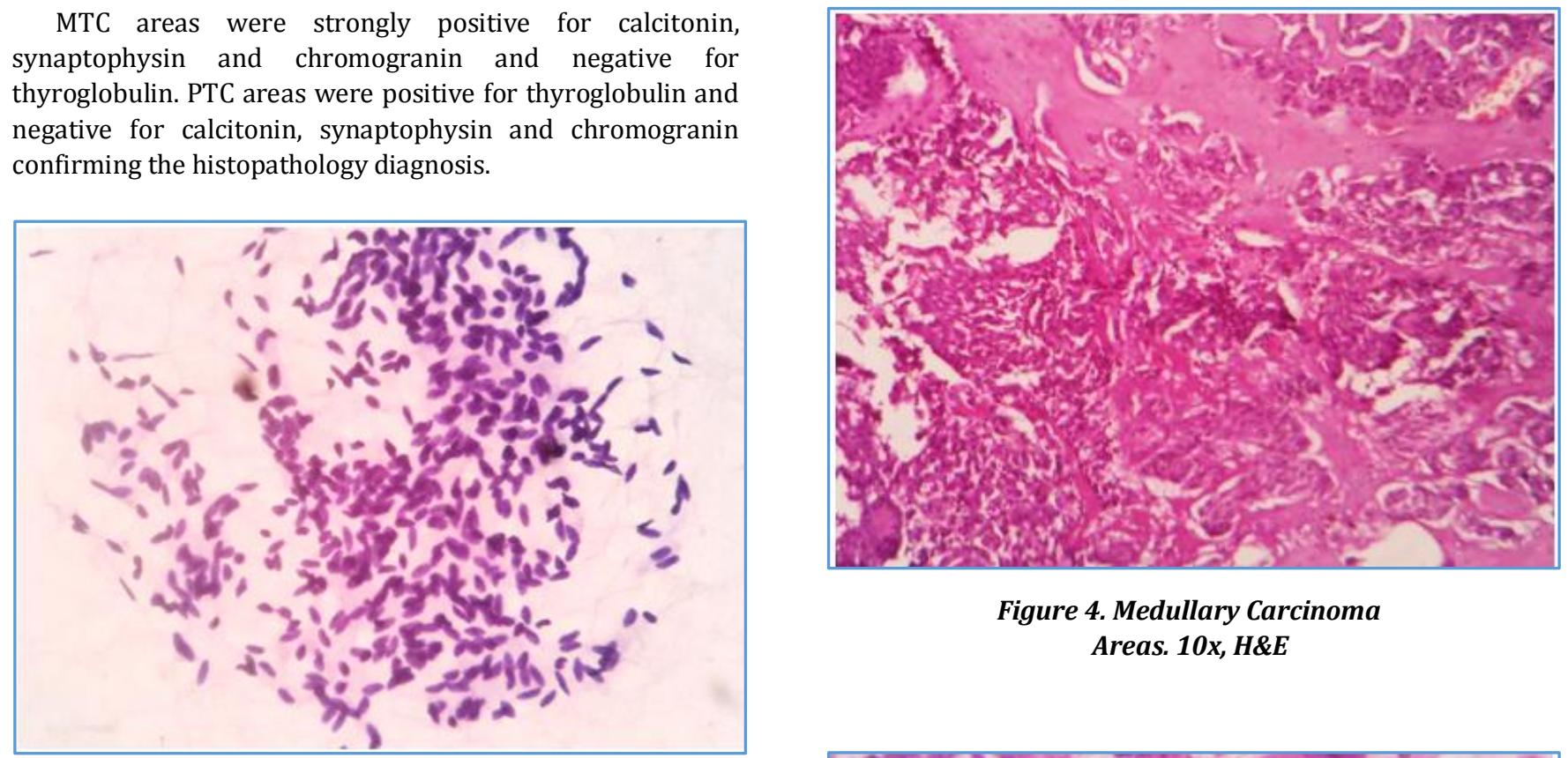

Figure 4. Medullary Carcinoma Areas. 10x, $\mathrm{H} \& E$

Figure 1. FNAC showing Clusters of Spindle Shaped Cells. 10x, H \& E
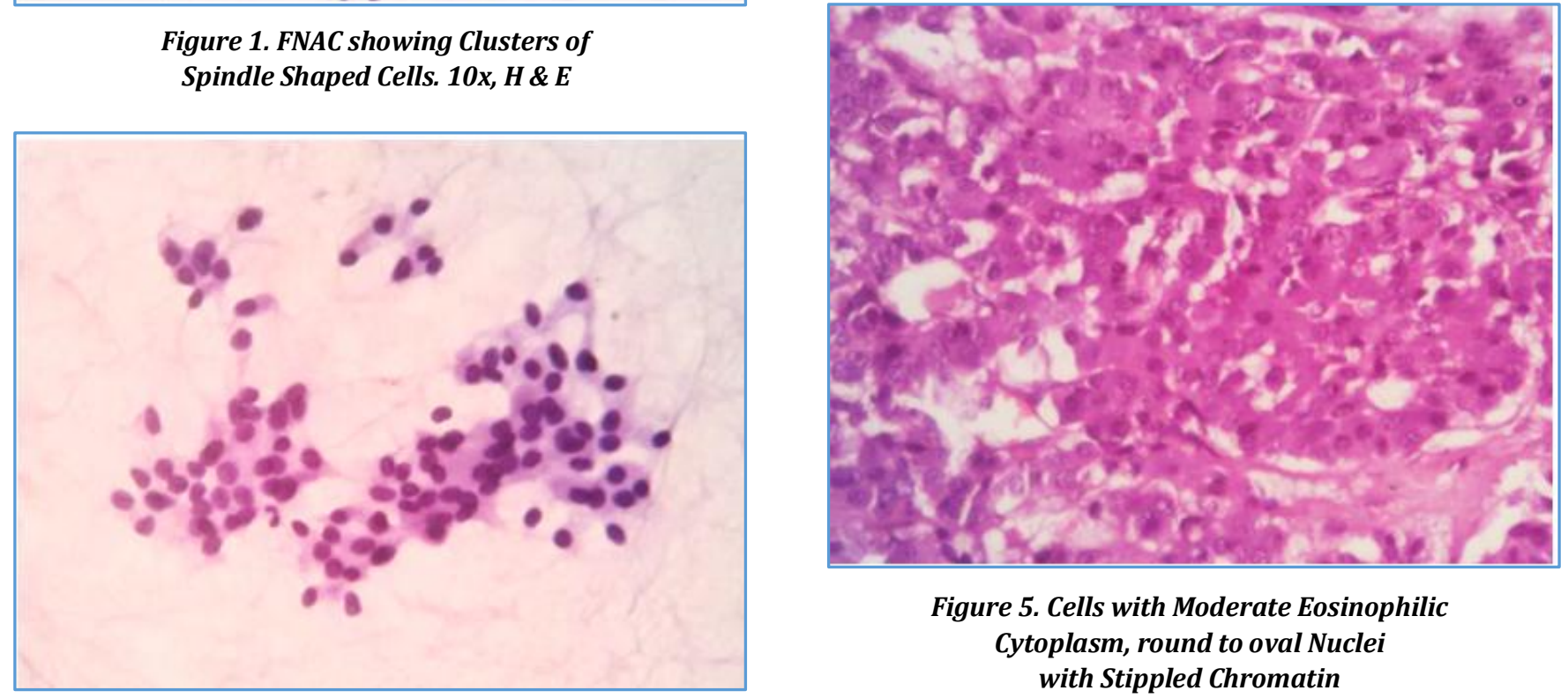

Figure 5. Cells with Moderate Eosinophilic Cytoplasm, round to oval Nuclei with Stippled Chromatin

Figure 2. FNAC showing Clusters of Plasmacytoid cells. 10x, H\&E

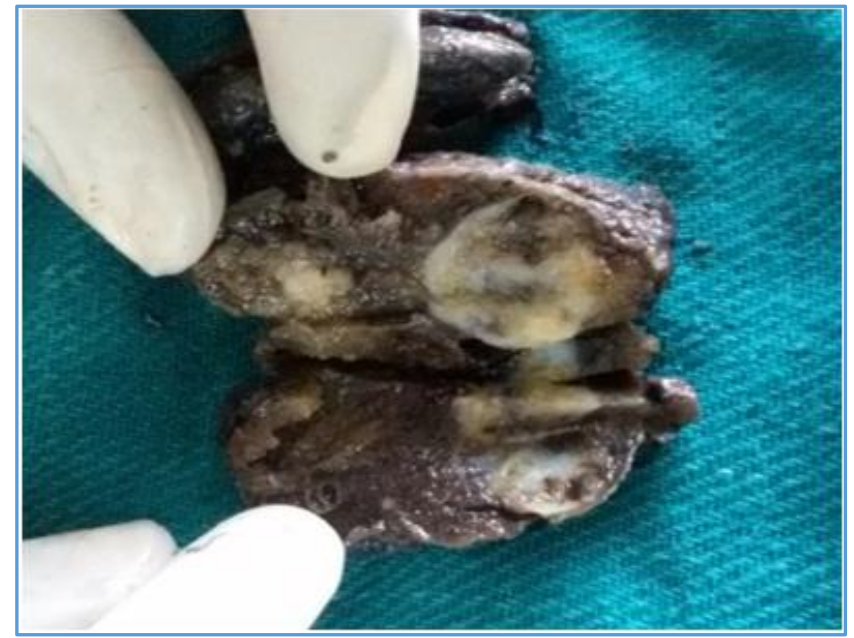

Figure 3. Gross picture of Thyroid Showing a Larger Nodule \& Another Smaller Nodule in Right Lobe

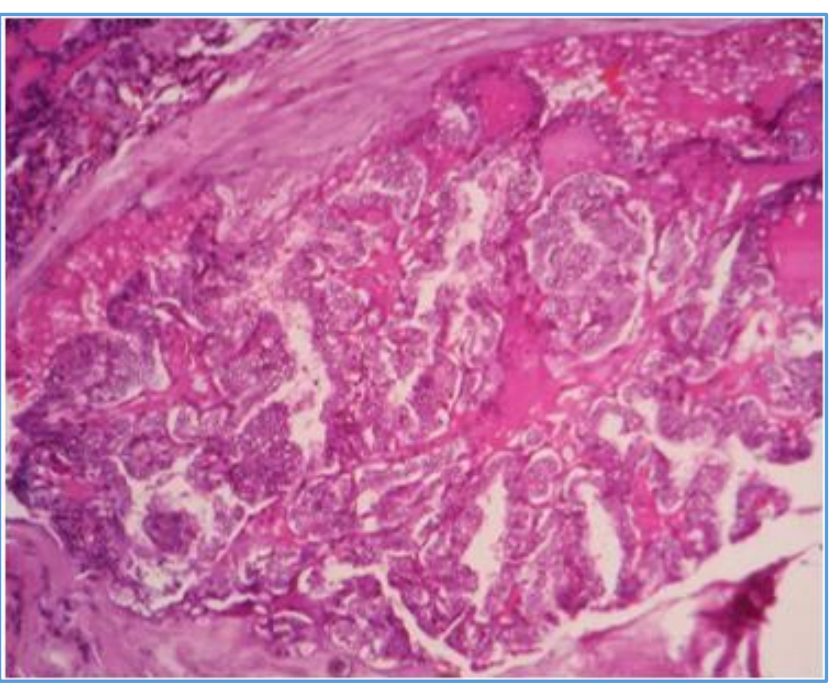

Figure 6. Papillary Carcinoma Areas. 10x, H\&E 


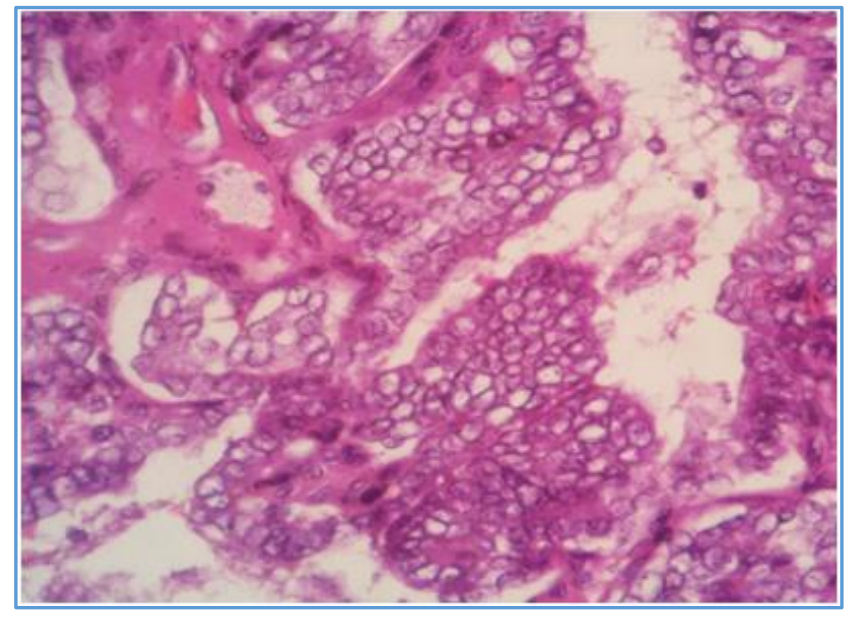

Figure 7. Papillary Carcinoma Areas showing Clearing of Nucleus Crowding and Overlapping

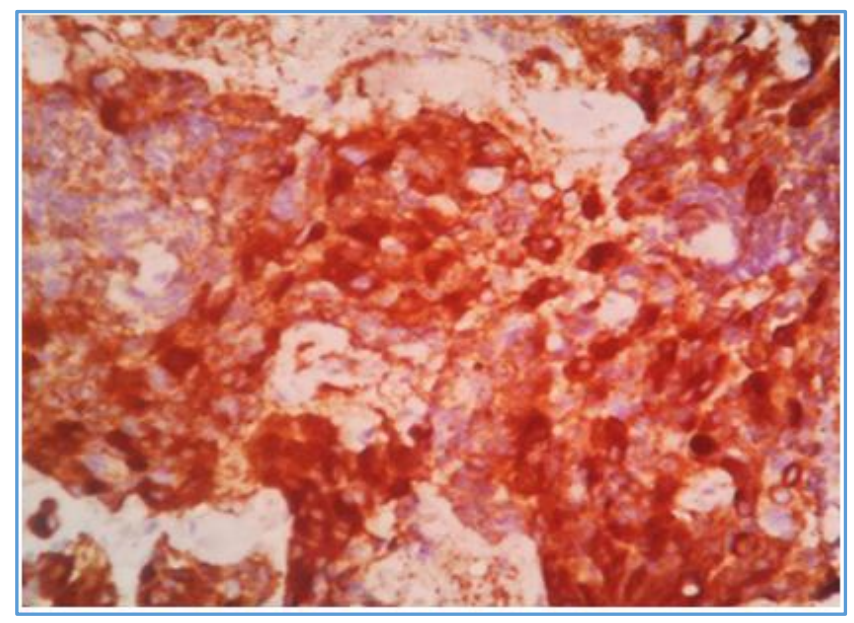

Figure 8. Medullary Carcinoma Area showing Positivity for Calcitonin

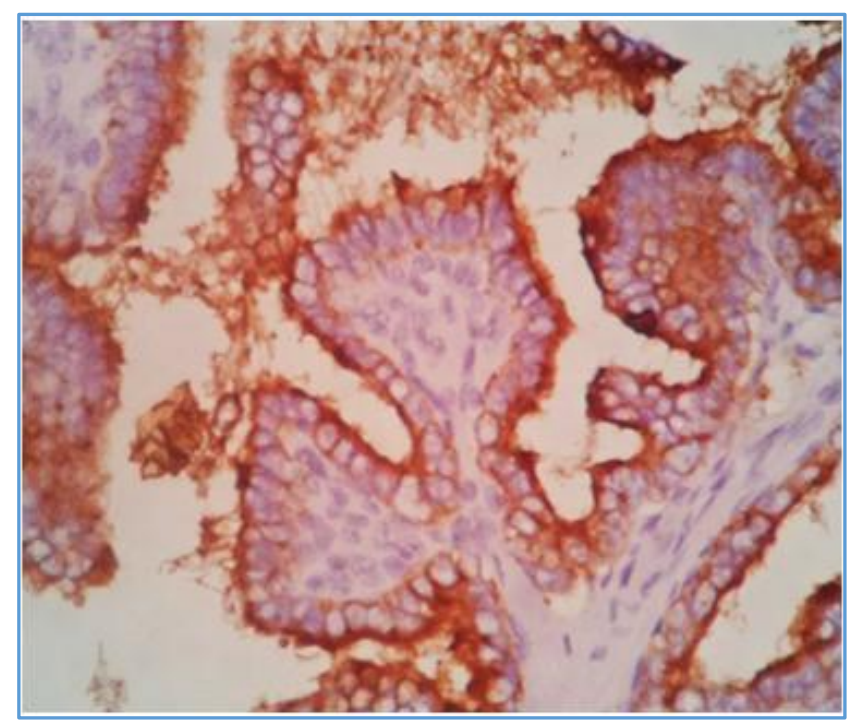

Figure 9. Papillary Carcinoma Area showing Positivity for Thyroglobulin

\section{DISCUSSION}

Traditionally, thyroid carcinomas are classified broadly into two groups based on their embryonic origins. The first group comprises of carcinomas arising from the follicular epithelial cells derived from the median endodermal anlage from tongue and includes the papillary and follicular carcinomas. The second group consists of carcinoma arising from parafollicular cells of ultimobranchial body of neural crest, which is the medullary carcinoma.

Papillary thyroid carcinoma is the most common histologic type of thyroid malignancies and accounts for about $75-80 \%$ of cases. They produce thyroid hormones and thyroglobulin. The molecular alterations associated with PTC are BRAF mutations (50\%), RET proto-oncogene rearrangements RET /PTC (10 - 30\%), RAS mutations (15\%). PTC is an indolent neoplasm with an excellent long term prognosis. Treatment is total thyroidectomy with or without radioiodine remnant ablation.

Medullary thyroid carcinoma is a malignant tumour with parafollicular $C$ cell differentiation. It accounts for $5-8 \%$ of all thyroid malignancies. The tumour cells secrete calcitonin and a variety of other peptide products. MTC is an aggressive type of thyroid carcinoma with a very low cure rate and high rates of relapse and mortality. It occurs as either sporadic $(80 \%)$ or hereditary (20\%) forms. The hereditary MTC may be familial or associated with MEN 2 syndromes. Activating point mutations in RET proto-oncogene and germline mutations in RAS are implicated in the pathogenesis of MTC. The mainstay of treatment is total thyroidectomy with neck node dissection. No effective adjuvant therapy is yet available.

In 1989, WHO classification system defined mixed medullary and follicular thyroid carcinomas (MMFTC) as a tumour showing morphological features of both MTC and carcinoma originating from follicular cells, including PTC. MMFTC constitute less than one percent of all thyroid cancers. Information regarding synchronous occurrence of MTC and PTC in same thyroid is mainly based on case reports and a few reviews in literature. Till 2005, only less than 20 cases of such concurrent occurrence were reported in literature. ${ }^{[4]}$ But since then there had been an increase in the number of reported cases. This can be attributed not only to the increase in prevalence of thyroid carcinomas, but also to the diagnostic advances made in the early detection of thyroid abnormalities. The increased use of ultrasonography and FNAC in thyroid diseases have contributed in early and effective diagnosis of these entities.

The differential diagnosis that should be considered is the papillary variant of MTC which may morphologically mimic the mixed MTC \& PTC.[5] Immunohistochemical (IHC) markers are important to differentiate between these two entities. The thyroglobulin positivity and calcitonin negativity in PTC areas support the diagnosis of co-existent MTC and PTC, whereas calcitonin positivity in PTC areas support papillary variant of MTC. Another differential diagnosis is MTC with normal or reactive thyroid follicle entrapment. Here, the typical nuclear features of PTC like nuclear clearing, overlapping, grooves and pseudoinclusions will be absent.

The first case of co-occurrence of MTC \& PTC was described by Lamberg et al in 1981.[6] Since then many cases were reported and studied at molecular levels to identify the possible pathogenesis of these tumours. 
The various hypotheses postulated[7] were 1). Common stem cell theory - suggests that both tumours arise from common uncommitted stem cells derived from ultimobranchial body. Somatic genetic analysis of both MTC and PTC has evidenced different mutation patterns which is against this theory. 2). Hostage hypothesis - proposed that non-neoplastic follicular cells are entrapped by MTC and they proliferate via trophic factor stimulation. Genetic alterations during follicular cell proliferation lead to neoplastic transformation. 3). Field effect theory - proposes common neoplastic stimuli resulting in simultaneous malignant transformation of follicular and parafollicular cells. 4). Collision theory - suggests that two independent tumours are located in the same thyroid by simple coincidence.

Most of the case reports and reviews have concluded that the synchronicity may be coincidental, because of lack of definite proof on molecular or genetic linked pathogenesis, thus supporting collision theory. In the last two decades, many authors have done genetic analysis of RET proto-oncogenes, but had conflicting results. Brauckhoff et al reported that a germline mutation of RET gene had a potential role in development of both MTC \& PTC. [8] Rossi et al in 2005 reported that both RET and BRAF genes had a role in the genesis of MTCPTC collision tumour. ${ }^{[4]}$ However, Vantyghem et al reported 17 cases of familial MTC-PTC, but none had RET defects. ${ }^{[9]}$ A few studies like Lax et al in 1994[10] and Shiroko et al in 2000[11] have reported that mixed MTC-PTC had arisen from a common stem cell, thus supporting the stem cell theory.

In our case, MTC and PTC presented as two distinct and well-defined tumour components separated by normal thyroid parenchyma. Moreover, IHC studies were conclusive of two separate tumours. Hence, the coexistence of MTC and PTC in this case may be coincidental as proposed by collision theory. Since both these tumours were confined to thyroid with no local extension or lymph node metastasis, the treatment is total thyroidectomy without any need for adjuvant therapy.

\section{CONCLUSION}

Synchronous occurrence of MTC and PTC is an extremely rare clinical entity and the surgeon and pathologist should be aware of such a possible coexistence. Many of the cases reported in literature as well as our present case are possibly due to a simple coincidence based on the evidences available in the literature. Further studies using larger number of cases are necessary to prove or disprove any possible genetic linkage for such synchronicity.

\section{REFERENCES}

1. Wong RL, Kazaure HS, Roman SA, et al. Simultaneous medullary and differentiated thyroid cancer: a population-level analysis of an increasingly common entity. Ann Surg Oncol 2012;19(8):2635-42.

2. Fugazzola L, Cerutti N, Mannavola $D$, et al. Multigenerational familial medullary thyroid cancer (FMTC): evidence for FMTC phenocopies and association with papillary thyroid cancer. Clin Endocrinol (Oxf) 2002;56(1):53-63.

3. Alavi SM, Azarpira N. Medullary and papillary carcinoma of thyroid gland occurring as a collision tumor with lymph node metastasis: a case report. J Med Case Rep 2011;5(1):590.

4. Rossi S, Fugazzola L, De Pasquale L, et al. Medullary and papillary carcinoma of the thyroid gland occurring as a collision tumour: report of three cases with molecular analysis and review of the literature. Endocr Relat Cancer 2005;12(2):281-9.

5. Jain M, Verma D, Thomas S, et al. Mixed medullarypapillary carcinoma thyroid: an uncommon variant of thyroid carcinoma. J Lab Physicians 2014;6(2):133-5.

6. Lamberg BA, Reissel P, Stenman S, et al. Concurrent medullary and papillary thyroid carcinoma in the same thyroid lobe and in siblings. Acta Med Scand 1981;209(5):421-4.

7. Gurkan E, Gurbuz Y, Tarkun I, et al. Mixed medullarypapillary carcinoma of the thyroid: report of two cases and review of the literature. Indian J Pathol Microbiol 2014;57(4):598-602.

8. Brauckhoff M, Gimm O, Hinze R, et al. Papillary thyroid carcinoma in patients with RET proto-oncogene germline mutation. Thyroid 2002;12(7):557-61.

9. Vantyghem MC, Pigny P, Leteurtre E, et al. Thyroid carcinomas involving follicular and parafollicular C cells: seventeen cases with characterization of RET oncogenic activation. Thyroid 2004;14(10):842-7.

10. Lax SF, Beham A, Kronberger-Schonecker D, et al. Coexistence of papillary and medullary carcinoma of the thyroid gland-mixed or collision tumour? Clinicopathological analysis of three cases. Virchows Arch 1994;424(4):441-7.

11. Shiroko T, Yakoo N, Okamoto K, et al. Mixed medullarypapillary carcinoma of the thyroid with lymph node metastases: report of a case. Surg Today 2001;31(4): 317-21. 